\title{
On the determination of the angular distance of the colours
}

\section{M.A. Rosenstiehl}

To cite this article: M.A. Rosenstiehl (1881) On the determination of the angular distance of the colours, Philosophical Magazine Series 5, 12:74, 228-230, DOI: 10.1080/14786448108627097

To link to this article: http://dx.doi.org/10.1080/14786448108627097

曲 Published online: 17 May 2010.

Submit your article to this journal $\sqsubset \pi$

Џ Article views: 2

Q View related articles $\asymp$ 
to be this difference of potential "corrected for the effect of the current passing (if appreciable)," the correction being the addition of the quantity $b \mathrm{C}=\frac{b}{b+\rho} \mathrm{E}$. This is obviously equivalent to defining the E.M.F. as $\left(\mathrm{V}-\mathrm{V}^{\prime}\right) \frac{b+\rho}{\rho}$.

I have the honour to be, Gentlemen, Your most obedient Servant,

Charles R. Alder Wright.

Chemical Laboratory, St. Mary's Hospital, W., August 3, 1881.

\section{Intelligence and Miscellaneous Articles.}

ON THE DETERMINATION OF THE ANGULAR DISTANCE OF THE

COLOURS. BY M. A. ROSENSTIEHL.

TWO series of experiments made upon a chromatic circle called my 1 attention to three colours appearing to fulfil special functions*. I was thus led to discuss Young's theoryt on the three fundamental sensations, and to establish two categories of non-complementary colours :- 1 , those which by their mixture in threes at equal intensity produce the sensation of white; 2 , those which produce that sensation outside of those proportions.

I have designated the first category by the name "triad;" and I remarked that from their definition it follows that, representing the table of colours by an equilateral triangle of which each vertex is occupied by one of the colours of a triad, the complementaries will be placed, in that construction, on the sides of the triangle and at the extremities of a straight line passing through the point of meeting of the median lines, while this regularity cannot exist for the second category of combinations of three colours.

The aim of the present note is to prove that the three colours above referred to, viz. orange, the third yellow-green, and the third blue, possess the characters of a triad.

By a first series of experiments I determine the pairs of complementary colours. By a second series I ascertain the proportions in which the three colours chosen must be mixed in order to obtain the sensation of white. By a third series I seek to reproduce a determined colour by the mixture of two or three colours: all these experiments are made with rotating disks; the measurement of the angles of the sectors gives the position of this colour on the sides of the triangle. In the calculations I do not take into consideration the white which is produced at the same time, but only the sensation of colour, my aim being to determine the proportion in which two different colour-sensations must be mixed to obtain a

- Phil. Mag. March and April 1881, vol xi. pp. 222, 305.

† Ibid. vol. xii. p. 78 . 
determined sensation of colour. The results of the three series of experiments are summed up in the following figure.

3rd yellow-green.

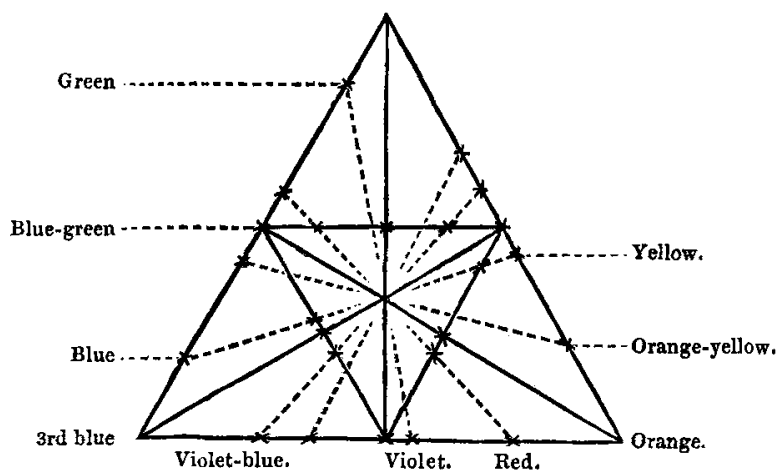

It is there seen that the straight lines which join the complementary colours intersect in a single point. Consequently the three separate colours form part of a triad; here is their position in the spectrum:-

$$
\begin{aligned}
& \text { Orange at } \ldots \ldots \ldots \ldots \ldots \\
& \text { Green (3rd yellow-green) at } \frac{3}{4} \text { from } \mathrm{C} \text { to } \mathrm{D} \text {. } \\
& \text { Blue (3rd blue) at....... } \mathrm{E} \text {. }
\end{aligned}
$$

I repeated the last two series of experiments, methodically replacing one of the three colours by another. I did the same with the three colours which Maxwell considers fundamental. None of these systems give regular results. Some colours which to the eye are very close are in them separated by large spaces, while some distinct colours are there brought very near together and almost blended, and the lines joining two complementary colours do not intersect in only one point.

The two propositions above formulated are therefore in accordance with experiment.

To complete the demonstration of the existence of the triads, it remains for me to prove that all the colours which occupy the vertices of an inseribed equilateral triangle possess the same properties.

I ascertained that it really is so by recommencing the same series of experiments with the triad formed by the first violet, the bluegreen, and a yellow comprised between the first and second yellows of my chromatic circle. These three colours are distant $120^{\circ}$ in the above figure. The results of the experiment are there indicated. It will be remarked, not only that the complementary colours are situated at the extremities of a straight line passing through the point of intersection of the median lines, but also that the new figure is exactly superposable to the first in what concerns the position of the intervening colours. We may conclude from this that the angular distance of the colours is the same, whichever may be the triad employed as starting-point.

Plil. Mag. S. 5. Vol. 12. No. 74. Sept. 1881. 
This angular distance is therefore determined once for all; but not so the form of the table of colours, which is independent of it, since the same result can be arrived at with an infinity of triads.

Which of the triads corresponds to the fundamental sensations? To answer this question we have no longer exact measures to guide us, but only eye-estimates. It may in reality be enunciated thus :Are the colours for which the angular distance is the same equidistant to sight? If the answer is yes, all the triads have the same value, and the table of the colours can be represented by a circle. If, on the contrary, there are colours equidistant to sight whose complementaries appear more distant from or nearer to one another, the table of the colours presents angles.

The study which I have previously made of the distribution of the complementary colours in a chromatic circle shows incontestably that all the colours comprised between the red and the orangeyellow have complementaries very near the blue-green, and those comprised between the blue and the violet-blue have their complementaries very close to the yellow. There are therefore vertices towards the violet-blue and the orange. The difference appears to me less accentuated for the third yellow-green.

However it may be with the form of the table of colours, its projection upon the sides of an equilateral triangle has permitted the determination of the exact angular distance of the colours-a result which had not yet been obtained, and which suffices to connect the facts at present known. The above diagram represents the law of the mixture of colours, and may be substituted with advantage for Newton's rule.

It has moreover an asthetic meaning: by the determination of the triads it gives facility for applying to arrangements of three colours the rules of harmony which I have given for the employment of complementary colours in decoration.-Comptes Rendus de l'Académie des Sciences, July 25, 1881, t. xciii. pp. 207-210.

\section{ON $\triangle$ METHOD FOR AMPLIFYING DISPLACEMENTS OF THE PLANE} OF POLARIZATION. BY M. H. BECQUEREL.

When monochromatic luminous rays pass through a half-wave crystal plate, the emergent rays are, as is known, rectilinearly polarized in a plane which, in regard to the axis of the crystal plate, is symmetric with the plane of polarization of the incident rays. This property of a half-wave plate can be utilized for doubling and trebling the measures of the displacements of the plane of polarization of light. This can be accomplished especially in the following manner:-

Having arranged an experiment for the purpose of measuring a rotation of the plane of polarization of light, the investigator commences by fixing with the greatest care, by means of the analyzer, the initial position of the plane of polarization of the incident rays. A half-wave plate is then interposed in front of the analyzer and rotated until the plane of polarization of the light-rays is not deflected by their passing through the plate. In this position the 